\title{
Salvage surgery after failure of non-surgical therapy for advanced head and neck cancer
}

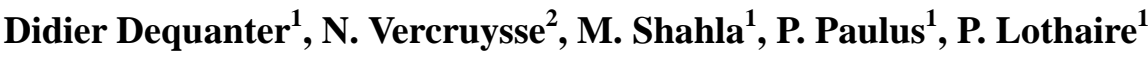 \\ ${ }^{1}$ Department of Head and Neck Surgery, CHU Charleroi, Montigny le Tilleul, Belgium; \\ ${ }^{2}$ Department of Plastic Surgery, CHU Charleroi, Montigny le Tilleul, Belgium. \\ Email: didier.dequanter@pandora.be
}

Received 25 July 2011; revised 2 September 2011; accepted 15 September 2011.

\begin{abstract}
Introduction: for organ and function preservation, chemoradiotherapy is gaining popularity for primary treatment of advanced head and neck cancer, reserveing surgery for salvage. Methods: Retrospective outcome analysis to determine the results of salvage surgery after failure of primary treatment of advanced head and neck cancer by chemoradiotherapy. 104 patients with advanced head and cancer were initially treated by chemoradiotherapy. Follow-up was evaluated in $\mathbf{2 7}$ patients undergoing salvage surgery for recurrent tumor (larynx $n=13$; oral cavity $n=9$; hypopharynx $n=5$ ). The initial tumor is stage $T 3$ in 11 cases and $T 4$ in 16 cases. 10 patients had primary tumors stage III and 17 patients had tumors stage IV. Results: One postoperative death occured following surgery. The overall incidence of complications was 9/ 27 (\%). Recurrent disease developed at the primary initially treated in 25 cases and in the neck in 2 cases after a mean follow-up of 11 months (3 - 136 months). After salvage surgery, loco-regional recurrence and/ or distant disease developed in 10/27 patients after a mean follow-up of 4 months. 6/10 (60\%) patients died after re-recurrence despite salvage chemotherapy. Conclusion: Salvage surgery after failure of initial chemoradiotherapy is burdened with high morbidity and bad oncological outcome. We demonstrated that it is difficult to salvage locally recurrent head and neck cancer especially at more advanced T-stages or when tumor recur. The limited effect of surgical salvage for recurrent tumor need to be addressed when choosing the initial treatment plan.
\end{abstract}

Keywords: Head and Neck Cancer; Salvage Surgery; Advanced Disease

\section{INTRODUCTION}

Chemoradiation therapy is gaining popularity for pri- mary treatment of advanced head and neck cancer, reserving surgery for salvage. Chemoradiotherapy is increasingly used for organ and function preservation. In a substantial amount of patients, chemoradiation failure is seen. Approximatively, $13 \%-24 \%$ of patients will, in reality, require salvage surgery [1].

Data about outcome after salvage surgery is necessary to assess its value as a second line tool. The two main aspects for an analysis concern the oncological outcome on one hand and the morbidity on the other hand. Both have been reported with broad variation in results among different groups [1-4].

This study aims to examine the outcome of salvage surgery as an additional treatment option in a determined group.

\section{METHODS}

104 patients initially treated by chemoradiotherapy were retrospectively reviewed in order to determine the clinical course of patients treated by salvage surgery after failure of primary treatment. In presence of a locoregional recurrence, the option of salvage surgery was analyzed. Absence of distant metastases and feasibility of complete surgical resection with acceptable postoperative functional outcome are premises for the indication of salvage surgery. In case of local recurrence, the surgical strategy depends on the result of microlaryngoscopy and CT scan. If possible, partial laryngectomy is taken in to consideration, otherwise total (pharyngo) laryngectomy is indicated. The neck was treated simultaneously by neck dissection of the levels II-V in all cases of local recurrence. In presence of a persisting neck mass and radiological suspicious lymph nodes, microlaryngoscopy and CT scan of the neck are performed in order to exclude oral, laryngeal or hypopharyngeal recurrence. Then, neck dissection of the levels II-V was performed based on the clinical suspicion and without further preoperative lymph node biopsy. The patients with previously untreated T3 and T4 head and neck tumor were 
treated for curative intent by chemoradiotherapy. Successful surgical salvage was defined as no evidence of recurrent cancer at 2 years after salvage surgery and continuously thereafter. All patients undergoing salvage surgery were analyzed for postoperative morbidity.

104 patients with advanced head and neck cancer received concomitant chemoradiotherapy (CRT) as an initial treatment. At the end of the follow-up, loco-regional recurrence was observed in 32 patients. 5 patients with non-responding tumors the proposal salvage surgery.

To determine the significance of clinical factors (such as age, gender, primary tumor site, TN stage, pTN stage on the recurrence, the chi-square test was used. A p < 0.05 was considered statistically significant.

\section{RESULTS}

Of the 104 patients, 27 patients with recurrent head and neck cancer tumor were reviewed. The primary site was the larynx in 13 cases, the oral cavity in 9 cases and the hypopharynx in 5 cases. The $\mathrm{T}$ stage at initial presentation was T3 in 11 cases, T4 in 16 cases. 10 patients were stage III and stage IV in 17 cases (Table 1). One postoperative death occured following salvage surgery. The patient died from hepatic disease without showing evidence of tumor.

Recurrence developed at the primary in 25 cases (larynx in 13 cases, or al cavity in 7 cases and hypopharynx in 5 cases) and at the neck in 2 cases after a mean follow-up of 11 months (3 - 136 months).

Total laryngectomy was needed in 11 cases, a partial laryngectomy in only 2 cases, a pharyngolaryngectomy in 3 cases, a buccopharyngectomy in 7 cases and a pharyngectomy in 2 cases. A radical neck dissection as salvage treatment was done in 2 cases. The overall incidence of complications was 9/27 (37.5\%). Among the 9 patients, eight patients (who underwent complete (pharyngo) laryngectomy developed a pharyngocutaneous fistula. In four cases, the fistula closed spontaneously. In 5

Table 1. Patient characteristics.

\begin{tabular}{cc}
\hline Characteristics & No. patients \\
\hline Gender Male/Female & $22 / 5$ \\
Age (years) & 8 \\
$30-50$ & 16 \\
$51-70$ & 3 \\
$71+$ & \\
Stage & 10 \\
III & 17 \\
IV & \\
Primary tumor location & 5 \\
Pyriform sinus & 13 \\
Larynx & 9 \\
Oral cavity & \\
\hline
\end{tabular}

cases, surgical closure of the fistula was needed.

The last patient developed a cervical abcess that resolved spontaneously.

After the salvage treatment, loco-regional recurrence and/or distant metastases developed in 10/27 (42\%) patients after a mean follow-up of 4 months. Recurrence was diagnosed in the neck and the primary site in 2 cases, in the neck alone in 3 cases, in the neck and with distant metastases in one case, at the primary site in 4 cases.

Among the 10 patients who developed recurrence after salvage surgery, 6 patients (60\%) died despite salvage chemotherapy.

\section{DISCUSSION}

Concomitant chemoradiation (CRT) is gaining popularity for primary treatment of advanced head and neck cancer, reserving surgery for salvage. However, it has been reported that the feasibility of salvages therapies following tumor recurrence is generally low. In his series, Taki et al. [5] reported that the local recurrence rate of hypopharyngeal cancer was $43.7 \%$ in patients with T2 T4 disease after treatment with RT (CT) but salvage surgery is often the only curative option for recurrent cancer.

Patients undergoing salvage surgery can be divided into two groups: patients with local recurrences on one hand and patients with persistent lymph node metastases but no evidence of persistent primary tumor on the other hand.

One the hand, surgical therapy of local recurrence requires radical removal with or without neck dissection. In our study, the tumor recurrence seemed suitable for partial laryngectomy in only two cases while in 11 cases complete laryngectomy was indicated. On one hand, this is due to the initially advanced tumor stage and one the other hand, the diagnosis of recurrence is delayed by the oedema occurring after irradiation.

However, in recent decades, partial laryngectomy has been used successfully in such patients $[3,4]$. There is evidence that salvage surgery following (chemo) radiotherapy for early stage laryngeal carcinoma gives better survival rates and enables more often partial resections than in the presence of advanced initial stages [5-9].

But considering the bad overall survival for salvage surgery in advanced laryngeal and hypopharyngeal cancer, total laryngectomy must be considered the main procedure for failure after CRT [10]. In our study, partial pharyngolaryngectomy with or without reconstruction was done for 11 patients. Of the 11 patients, recurrences were seen in patients with tumors of the pyriform sinus. We think that advanced tumor stage with metastatic spread, hypopharyngeal localisation and previous radical radiochemotherapy have contributed significantly to the unsatisfying results of salvage surgery. Thereby, an earlier detection of persistent and growing tumor tissue 
after completion of (chemo) radiotherapy might improve the prognosis of salvage surgery.

Simultaneous neck dissection has been recommended in the literature because of neck recurrence in previously unsuspicious neck [11,12]. However, the oncological value of simultaneous neck dissection in the absence of suspect lymph nodes has also been questioned [13]. Temam et al. [2] found lymph nodes metastases only in one of the 30 patients undergoing salvage surgery for local recurrence of initially irradiated carcinoma of the head and neck with cN0 necks. They therefore recommend limitation of neck dissection according to the initial $\mathrm{N}$ status. In our series, mostly, the $\mathrm{N}$ status after salvage surgery for local recurrences including neck dissection was congruent with the $\mathrm{N}$ status before chemoradiotherapy in cases while in one case an initial cN2b stage turned into a pN0 stage after salvage surgery. This data suggest a coherence of initial clinical lymph nodes status with that in case of local recurrence after CRT in most cases. Thus, less radical neck surgery in absence of initial and preoperative lymph nodes metastases has to be discussed.

On the other hand, neck dissection (ND) for persistent cervical lymph nodes may be judged an overtreatment for the majority of our patients when no vital tumor could be found in the neck specimen. None of our patients with N0 neck developed regional recurrence during the follow-up. The indication for neck dissection (ND) is based upon the clinical suspicion the main problem in preoperative verification of a metastasis relies in the difficulty to distinguish scarred lymph node tissue from nodes containing vital tumor cells. Patients with histologically confirmed persistent cervical metastases had a high tendency of further regional recurrences and distant metastasis. In the literature, neck dissection after chemoradiotherapy yielded unfavourable results for histologically proven $\mathrm{N}+$ situations. On the other hand, ND seems to be inappropiate if no vital tumor tissue is detected after excision of suspicious residual nodes. Some authors advocate a wait and see policy for persistent lymph nodes in head an neck cancer as long as a decrease in size can be demonstrated after chemoradiotherapy [14]. Others are in favor of an immediate ND after RCHT for all patient with initial N2-N3 [15] or only in N3 [16-19] neck disease even in presence of complete response. We believe that salvage ND for suspicion of residual disease is of low morbidity and should therefore be recommended in regard to individual tumor control. Performance of radical instead of selective ND must be discussed in this context and should examined further.

But not only the type of salvage surgery must be discussed, but also the selection of patients candidate to salvage surgery is an important issue.

In our series, 10 of the 27 patients with advanced head and neck cancer presented locoregional recurrence and/ or distant metastases.

Most patients (8/10) who developed a recurrence had a tumor staged T4. Clearly, the tumor extension should be an important factor in the selection of patients candidate to salvage surgery. Indeed, Schwartz et al. in his series [20], showed that individuals with stage I-II primary tumors had significantly improved salvage time and total survival time compared with those with stage III-IV primary tumors ( $p<0.005$ and $\mathrm{p}<0.001)$. Conversely, the stage of the recurrent tumor was not predicttive of either improved salvage time and total survival time. Patients who underwent salvage surgery had significantly improved salvage time and total survival time compared with those who received chemotherapy and/or radiation therapy ( $<0.001$ and $\mathrm{p}<0.002$ ). Neither the stage of the primary or recurrent tumors nor the type of salvage treatment received significantly correlated with an improved cure rate. Patients most likely to benefit from retreatment are those who have primary tumors stage I-II, recur greater than 6 months after theri initial treatment and develop recurrences that are amenable to salvage surgery. In his series, Gleich [21] confirmed the limited potential for survival in patients who have a recurrence after treatment for advanced (T3 or T4) primary site head and neck cancer. 48 patients underwent salvage therapy for recurrence. The median time to recurrence was 14 months and the median survival time was 26.2 months. Among the 28 patients treated for primary site recurrence, the mean time for recurrence was 12.6 months and the mean survival time was 27.3 months. Only the 5 of the 28 patients had prolonged survival. The stage of recurrent disease did not influence the outcome. Among the 20 patients treated for neck recurrence, the mean time to recurrence was 14 months and the mean time survival time was 25 months. Six of the 20 patients had prolonged survival.

In our study, among the patients treated for neck recurrence, the mean time to recurrence was 8 months and the mean time survival time was 18 months.

In his series, Kim et al. [22] determined the factors predicting the outcome after salvage surgery with microvascular flap reconstruction for recurrent squamous cell cancer of the head and neck. They concluded that patients with early cancers are the best candidates for salvage surgery but patients with $\mathrm{T} 3$ and $\mathrm{T} 4$ and who continue to smoke after initial diagnosis and treatment are poor candidates to undergo salvage surgery.

For Wong et al. [23], surgical salvage is only feasible for early recurrent tumor. In this series, published by Goodwin et al. [24], median disease free survival was 17.9 months in 109 patients, and this correlated strongly with recurrent stage, weakly with recurrent site, and not at all with time to presalvage recurrence. 
Therefore, Yom et al. [25] proposed for patients with T3 T4 tumors a planned clinical, radiographic, and pathologic restaging at 1 to 2 months after initial treatment. This practice produces improved overall local control and survival rates compared with the literature reports for delayed attempted salvage with timing base don the findings of routine clinical surveillance.

In addition, the chance for salvage surgery was more limited in patients with hypopharyngeal cancers. In this series [5], when the recurrence became unequivocal, most T3 and T4 patients were considered unsuitable to receive surgical salvage. Only two patients of 18 patients (11\%) who experienced local recurrence achieved long term survival after surgical salvage. All T4 patients died within 19 months after the local recurrence was detected. By contrast, laryngeal cancer offers more chance to undergo salvage surgery for recurrence [26,27].

Finally, some studies report an acceptable frequency of postoperative complications such as pharyngo-cutaneous fistulas after CRT $[4,26]$.

However, some authors report that preoperative chemoradiotherapy as a cause of increased postoperative morbidity in laryngeal and hypopharyngeal surgery as compared to the primary surgery has been postulated [1, 14,28].

Clark et al. [29] showed that irradiation increased the incidence of postoperative pharyngo-cutaneous fistula from $24 \%$ to $38 \%$ while Wakisaka [30] et al. noted that although the frequency of pharyngocutaneous fistula after RT or CRT was not high, fistula closure tended to be delayed. In their series, Taki et al. [5] experienced severe postoperative complications including pharyngocutaneous fistulas in $25 \%$ of patients who underwent laryngopharyngectomy. Such complications could severely diminish the quality of life for patients.

However, the frequency of published pharyngocutaneous fistulas in salvage surgery ranges from $14 \%$ to $92 \%$ [28] depending the applied dose [31] the tumor stage and localisation [14] and time from initial therapy [28]. As a comparison, pharyngocutaneous fistulas after laryngectomy without previous therapy occurred in about $11 \%$ for hypopharyngeal [32] and in 4\% for laryngeal carcinomas [33].

However, salvage surgery can be performed with acceptable rate of postoperative complications. In his series, Agra et al. [34] after analyzing the frequency and risk factors for postoperative complications after salvage surgery for recurrent head and neck cancers demonstrated that salvage surgery can be performed with acceptable rates of complications Post operative complications occurred in 66 patients (53.2\%). 53 patients (42.7\%) had minor complications and 23 patients (18.5\%) had major ones. There were 4 postoperative deaths (3.2\%)
The major factor associated with the overall occurrence of postoperative complications was the clinical stage of the recurrent tumor $(p=0.02)$ the occurrence of minor complications correlated with the previous treated site, with complications occurring more often in patients undergoing locoregional versus local treatment $(p=0.004)$ major complications were associated with the time between initial treatment and salvage surgery $(p=0.05)$.

Although most patients had good functional outcome only a select group of patients with recurrent SCC achieved long term survival after salvage surgery. In the literature, only a few articles have been published that discuss the treatment of patients with rerecurrence.

In our series, rerecurrence was diagnosed in 10 of the 24 patients. After a short follow up, 6 of the 10 patients died.

Patients with rerecurrence seems not to be candidate for salvage treatment and are at a high risk for death.

Indeed, patients with rerecurrence who have undergone all modalities of therapy, the chances for salvage are limited.

\section{REFERENCES}

[1] Ganly, I., Patel, S.G., Matsuo, J., et al. (2006) Results of surgical salvage after failure of definitive radiation therapy for early-stage squamous cell carcinoma of the glottic larynx. Archives of Otolaryngology-Head \& Neck Surgery, 132, 59-66. doi:10.1001/archotol.132.1.59

[2] Tenam, S., Koka, V. and Mamelle, G. (2005) Treatment of the N0 neck during salvage surgery after radiotherapy of head and neck squamous cell carcinoma. Head Neck, 27, 653-658. doi:10.1002/hed.20234

[3] Rickey, L.M., Shores, C.G., George, J., et al. (2007) The effectiveness of salvage surgery after failure of primary concomitant chemoradiation in head and neck cancer. Otolaryngology-Head and Neck Surgery, 136, 98-103. doi:10.1016/j.otohns.2006.06.1267

[4] Lee, S.C., Shores, C.G. and Weissler, M.C. (2008) Salvage surgery after failed primary concomitant chemoradiation. Current Opinion in Otolaryngology \& Head and Neck Surgery, 16, 135-140. doi:10.1097/MOO.0b013e3282f495b6

[5] Taki, S., Homma, A., Oridate, N., Suzuki, S., et al. (2010) Salvage surgery for local recurrence after chemoradiotherapy or radiotherapy in hypopharyngeal cancer patients. European Federation of Oto-Laryngological, 267, 17651769.

[6] Spriano, G., Pellini, R. and Romazno, G. (2002) Supracricoid partial laryngectomy as salvage surgery after radiation failure. Head Neck, 24, 759-765. doi:10.1002/hed.10117

[7] Nibu, K., Kamata, S. and Kawabata, K. (1997) Partial laryngectomy in the treatment of radiation failure of early carcinoma. Head Neck, 19, 116-120. doi:10.1002/(SICI)1097-0347(199703)19:2<116::AID-H ED5>3.0.CO;2-7

[8] Jorgensen, K., Godballe, C. and Hansen, O. (2002) Can- 
cer of the larynx-treatment results after primary radiotherapy with salvage surgery in a series of 1005 patients. Acta Oncologica, 41, 69-76. doi:10.1080/028418602317314091

[9] Marchese-Ragona, R., Marioni, G. and Chiarello, G. (2005). Supracricoid laryngectomy with cricohyoidopexy for recurrence of early glottic carcinoma after irradiation: Long term oncological and functional results. Acta Otolaryngol, 125, 91-95. doi:10.1080/00016480410017927

[10] Rodriguez-Cuevas, S., Labastida, S., Gonzalez, D., et al. (1998) Partial laryngectomy as salvage surgery for radiation failures in T1-T2 laryngeal cancer. Head Neck, 20, 630633.

doi:10.1002/(SICI)1097-0347(199810)20:7<630::AID-H ED9>3.0.CO;2-K

[11] Makeieff, M., Venegoni, D., Mercante, G., et al. (2005) Supracrociod partial laryngectomies after failures of radiation therapy. Laryngoscope, 115, 353-357. doi:10.1097/01.mlg.0000154751.86431.41

[12] Holsinger, F.C., Funk, E., Roberts, D.B., et al. (2006) Conservation laryngeal surgery versus total laryngectomy for radiation failure in laryngeal cancer. Head Neck, 28, 779-784. doi:10.1002/hed.20415

[13] Shamboul, K., Doyle-Kelly, W. and Bailey, D. (1984) Results of salvage surgery following radical radiotherapy for laryngeal carcinoma. Journal of Laryngology \& Otology, 98, 905-907. doi:10.1017/S0022215100147681

[14] Kraus, D.H., Pfister, D.G. and Harrsion, L.B. (1995) Salvage laryngectomy for unsuccessful larynx preservation therapy. Annals of Otology, Rhinology, and Laryngology, 104, 936-941.

[15] Yao, M., Roebuck, J.C. and Holsinger, F.C. (2005) Elective neck dissection during salvage laryngectomy. American Journal of Otolaryngology, 26, 388-392. doi:10.1016/j.amjoto.2005.05.002

[16] Farrag, T.Y., Lin, F.R. and Cummings, C.W. (2006) Neck management in patients undergoing postradiotherapy salvage laryngeal surgery for recurrent/persistent laryngeal cancer. Laryngoscope, 116, 1864-1866. doi:10.1097/01.mlg.0000234917.08822.cd

[17] Homma, A., Furuta, Y. and Oridate, N. (2006) Watch and see policy for the clinically positive neck in head and neck cancer treated with chemoradiotherapy. International Journal of Clinical Oncology, 11, 441-448. doi:10.1007/s10147-006-0603-4

[18] Dequanter, D., Lothaire, P. and Awada, A. (2006) Does clinical and radiological response predict complete tumor control in N2-N3 squamous cell head and neck cancer after non operative management of the neck? Acta Otolaryngol, 126, 1225-1228. doi:10.1080/00016480600818088

[19] Goguen, L.A., Posner, M.R. and Tishler, R.B. (2006) Examining the need for neck dissection in the era of chemoradiation therapy for advanced head and neck cancer. Archives of Otolaryngology_Head \& Neck Surgery, 132, 526-531. doi:10.1001/archotol.132.5.526

[20] Schwartz, G.J., Mehta, R.H., Wenig, B.L., et al. (2000) Salvage treatment for recurrent squamous cell carcinoma of the oral cavity. Head Neck, 22, 34-41. doi:10.1002/(SICI)1097-0347(200001)22:1<34::AID-HE D6>3.0.CO;2-3
[21] Gleich, L.L., Ryzenman, J., Gluckman, J.L., et al. (2004) Recurrent advanced (T3-T4) head and neck squamous cell carcinoma: Is salvage possible? Archives of Otolaryngology-Head \& Neck Surgery, 130, 35-38. doi:10.1001/archotol.130.1.35

[22] Kim, A.J., Suh, J.D., Sercarz, J.A., et al. (2007) Salvage surgery with free flap reconstruction: Factors affecting outcome after treatment of recurrent head and neck squamous carcinoma. Laryngoscope, 117, 1019-1023. doi:10.1097/MLG.0b013e3180536705

[23] Wong, L.Y., Wei, W.I., Lam, L.K., et al. (2003) Salvage recurrent head and neck squamous cell carcinoma after primary curative surgery. Head Neck, 25, 953-959. doi:10.1002/hed.10310

[24] Goodwin, W.J. (2000) Salvage surgery for patients wit recurrent squamous cell carcinoma of the upper digestive tract: When do the ends justify the means? Laryngoscope, 110, 1-18. doi:10.1097/00005537-200003001-00001

[25] Yom, S.S., Machtay, M., Biel, M.A., et al. (2005) Survival impact of planned restaging and early surgical salvage following definitive chemoradiation for locally advanced squamous cell carcinomas of the oropharynx and hypopharynx. American Journal of Clinical Oncology, 28, 385-392. doi:10.1097/01.coc.0000162422.92095.9e

[26] Leon, X., Quer, M., Orus, C., et al. (2001) Results of salvage surgery for local or regional recurrence after larynx preservation with induction chemotherapy and radiotherapy. Head Neck, 23, 733-738. doi:10.1002/hed.1104

[27] Stoeckli, S.P., Pawlik, A.B., Lipp, M., et al. (2000) Salvage surgery after failure of nonsurgical therapy for carcinoma of the larynx and hypopharynx. Archives of Otolaryngology_Head \& Neck Surgery, 126, 1473-1477.

[28] Lavertu, P., Bonafede, J.P. and Adelstein, D.J. (1998) Comparison of surgical complications after organ preservation therapy in patients with stage III or IV squamous cell head and neck cancer. Archives of OtolaryngologyHead \& Neck Surgery, 124,401-406

[29] Clark, J.R., De Almeida, J., Gilbert, R., et al. (2004) Primary and salvage (hypo) pharyngectomy: Analysis and outcome. Head Neck, 26, 272-277.

[30] Wakisaka, N., Murono, S.K., et al. (2008) Postoperative pharyngocutaneous fistula after laryngectomy. Auris Nasus Larynx, 35, 203-208. doi:10.1016/j.anl.2007.06.002

[31] Johansen, L.V., Overgaard, J. and Elbrond, O. (1988) Pharyngocutaneous fistulae after laryngectomy: Influence of previous radiotherapy and prophylactic metronidazole. Cancer, 61, 673-678. doi:10.1002/1097-0142(19880215)61:4<673::AID-CNC R2820610410>3.0.CO;2-C

[32] Dedo, D.D., Alonso, W.A. and Ogura, J.H. (1975) Incidence, predisposing factors and outcome of pharyngocutaneous fisutlas complicating head and neck surgery. Annals of Otology, Rhinology, and Laryngology, 84, 833-840.

[33] McCombe, A.W. and Jones, A.S. (1993) Radiotherapy and complications of laryngectomy. Journal of Laryngology \& Otology, 107, 130-132. doi:10.1017/S0022215100122406

[34] Agra, I.M., Carvalho, A.L., Pontes, E., et al. (2003) 
Postoperative complications after en bloc salvage surgery for head and neck. Archives of Otolaryngology-Head \&
Neck Surgery, 129, 1317-1321.

doi:10.1001/archotol.129.12.1317 